\title{
Impact of Metal Crystallinity-related Morphologies on the Sensing Performance of Plasmonic Nanohole Arrays
}

Received 00th January 20xx, Accepted 00th January 20xx DOI: $10.1039 / x 0 x x 00000 x$

\begin{abstract}
Mansoor Ali Khan, ${ }^{a}$ Ying Zhu, ${ }^{* a}$ Yin Yao, ${ }^{\text {}}$ Pengfei Zhang, ${ }^{c}$ Arti Agrawal, ${ }^{\text {d }}$ and Peter Reece, ${ }^{*} \mathrm{e}$
Plasmonic nanohole arrays has attracted tremendous attention in biosensing applications because of the flexibility in the optical signature design, high multiplexing capabilities, simple optical alignment setup, and high sensitivity. The quality of the metal film, including metal crystallinity and surface roughness plays an important role for determining the sensing performance because the interaction between free electrons in metal and incident light is strongly influenced by the metal surface morphology. We systematically investigated the influence of metal crystallinity-related morphologies on the sensing performance of plasmonic nanohole arrays after different metal deposition processes. We utilised several non-destructive nanoscale surface characterisations to provide a quantitative and comparative analysis of Au quality of the fabricated sensor. We find empirically how surface roughness and grain sizes influence the permitivity of the Au film and thus the sensitivity of the fabricated sensor. At last we confirm that depositions conditions that yield both a low surface roughness and large metal grain sizes improve the sensitivity of the plasmonic sensor.
\end{abstract}

\section{Introduction}

Optical biosensors are promising tools for the detection and analysis of disease biomarkers, environmental pollutants, virucidal drug evaluation for the applications such as clinical diagnosis, environmental monitoring and drug discovery..$^{1-3}$ In optical biosensors, plasmonic sensors are mostly used because of their merits such as wide dynamic detection range, highly developed surface chemistry, label-free and non-destructive detection towards a wide-range of biological samples, and lacking of electromagnetic interferences or complex sample conductivity requirements. ${ }^{4,5}$

The principle of plasmonic sensors is based on the coupling of incident plane waves to surface bound free electron oscillations, known as surface plasmons. Resonant coupling conditions (surface plasmon resonance, SPR) are dependent on momentum matching of incident optical to plasmon modes, which are sensitive to the geometry and local dielectric environment. ${ }^{6-8}$ To accomplish this, various methodologies such as prism couplers, nanoparticles and grating structures have been presented to satisfy momentum-matching conditions. Commercial SPR platforms using planar gold surface, such as

a. St George and Sutherland Clinical School, UNSW Sydney, NSW 2052 Australia. Email:ying.zhu@unsw.edu.au

b. Electron Microscope Unit, UNSW Sydney, NSW 2052 Australia

c. School of Photovoltaic and Renewable Energy Engineering, UNSW Sydney, NSW 2052 Australia

d. School of Electrical and Data Engineering, University of Technology Sydney, NSW 2007, Australia

e. School of Physics, UNSW Sydney, NSW 2052 Australia. Email: p.reece@unsw.edu.au

Electronic Supplementary Information (ESI) available. See DOI: $10.1039 / x 0 \times x 00000 x$
Biarcore $^{\mathrm{TM}}$ systems, have been widely used for real-time protein-protein binding based on prism-coupling-based Kretschmann configuration and refractive index sensing. ${ }^{7,} 8$ However, their bulky, expensive and complex alignment setups have limited their use in applications such as clinical diagnosis. On the other hand, metallic nanoparticles exhibiting localised SPR (LSPR) have been used for plasmonic sensing. ${ }^{9-11}$ However, LSPR has drawbacks in the detection of larger molecules binding or multiple layers away from surface because the electromagnetic field is confined to the metal surface with a decay length 40-50 times shorter than that of SPR-based detection systems. ${ }^{9}$ Hybrid plasmonic structures including grating structures such as nanoholes, nanopillars, bumps, and groves on flat metallic surfaces have been introduced to modulate the momentum of incoming photons and generate both localized and propagating surface plasmon resonance. ${ }^{11,12}$ Such structures represent a more robust sensing platform for point-of-care biomedical applications. ${ }^{13}, 14$

In context of grating structures, Ebbesen and co-workers reported an interesting fact that the transmission of light through an array of subwavelength nanostructured metallic surfaces, i.e. a periodic nanohole array (NHA) smaller than the wavelength of incident light, exhibits strong optical transmission properties at a particular wavelength than predicted by the classical theory. ${ }^{15}$ This extraordinary optical transmission (EOT) feature of NHA has attracted tremendous attention in biosensing applications because of the flexibility in the optical signature design, high multiplexing capabilities, simple optical alignment setup, and high sensitivity. ${ }^{16-18}$ Furthermore, such platform can be easily integrated with microfluidic devices for point-of-care devices. ${ }^{19,} 20$ Thus, NHA structures is a promising smart sensor tool for today's on-chip 
detection of biomolecular binding, drug discovery and clinical diagnosis. ${ }^{13}$

It has been reported widely that the shape of EOT (can be measured in the NHA transmission/reflection spectrum) can be adjusted by tuning the dimension of nanoholes (i.e. hole size and periodicity) and the dielectric properties of the metal. ${ }^{21-23}$ Gold has been the primary material in most plasmonic devices demonstrated to date due to its chemical stability and easy surface chemistry strategies (e.g. thiol-Au interaction). Besides the nanohole geometry and the dielectric properties of the metal, the quality of the metal film also plays an important role for the sensing performance including optical signature and sensitivity. ${ }^{24-26}$ The gold surface morphology, including crystallinity and surface roughness, are among the practical criteria to analyse the quality of metal film. Because surface plasmons exist very close to the interface, they are extremely sensitive to surface inhomogeneities, which can cause absorption, scattering and limited propagation. ${ }^{27}$ Rougher surface and polycrystallinity lead to a stronger damping in the localised surface plasmon modes due to increased electron scattering rates. ${ }^{25}$ The conduction-electron mean free path in bulk gold is $30-40 \mathrm{~nm} .{ }^{28,29}$ Smaller crystalline size than this leads to an increase in the frequency of electron-surface collisions and more electrons scattering events and damping. In addition, the surface roughness and grain boundaries can also enhance electron-defect scattering. Ideally, single crystalline with ultrasmooth surface can provide high-quality plasmonic materials because it will provide less scattering or diffusion paths for incident photons and resulting electron oscillation, as shown by Nagpal et al.. ${ }^{26}$ However, this method is not practical for most standard fabrication processes.

High-quality metal deposition methods can help minimise contributions of these non-radiative decay channels. ${ }^{30}$ Several studies have been carried out to investigate the influence of metal crystallinity and roughness on other types of optical devices. Wood et al. investigated the influence of silver grain size, roughness and grating profiles of silver gratings for their fluorescence enhancement capabilities and achieved an enhancement up to $116 \times$ compared to dye-coated glass slides. ${ }^{31}$ Leong et al. investigated the effect of surface morphology on the optical properties in a metal-dielectric-metal thin film as colour filters. They found an empirical relationship between the optical properties and the film structural properties. ${ }^{24}$ Very few studies have been dedicated to plasmonic NHA sensors. Zhang et al. utilised thermal annealing to improve the surface quality of gold film with a smoother surface and increase the surface sensitivity of NHA sensors by 2.5-3 times compared to nonannealed sensor. ${ }^{27}$ Therefore, there is an unmet need to analyse the quality of metal film using both quantitative and qualitative characterisations and correlate them with the sensing performance of plasmonic NHA sensors to guide the future of sensor design.

For the first time, we investigated the influence of metal crystallinity-related morphologies, particularly grain size and surface roughness, on the sensing performance of plasmonic NHA using different metal deposition processes. The different deposition methods gave us a series conditions for the variation in grain size and surface roughness, which allows us to systematically discuss their influence on the sensor performance. We utilise several non-destructive nanoscale surface characterisations including scanning electron microscope (SEM), atomic force microscopy (AFM), and electron backscatter diffraction (EBSD), which provide us a quantitative and comparative analysis of the Au quality of our fabricated NHA sensor. SEM shows the hole dimensions of an NHA sample such as hole shape, dimension and periodicity; AFM reveals the surface topography in more precise features such as surface roughness; and EBSD technique provides grain morphology in terms of grain size, grain boundaries, texture, crystallographic orientation. We establish empirical relationships between the optical sensitivity and key Au film structural properties (i.e. surface roughness and grain size). By quantitively correlating these results, we obtained a unique insight into the quality of the deposited Au film and established an empirical criterion for evaluating and optimising our fabricated NHA plasmonic sensor for better sensing performance.

\section{Results and discussion}

The procedure for fabricating an NHA structure is illustrated in Scheme 1. The NHA geometry we used are through-hole structures on a suspended SiN film. This arrangement was chosen for several reasons. First, an unique advantage of through-hole NHA compared to other plasmonic sensors is that

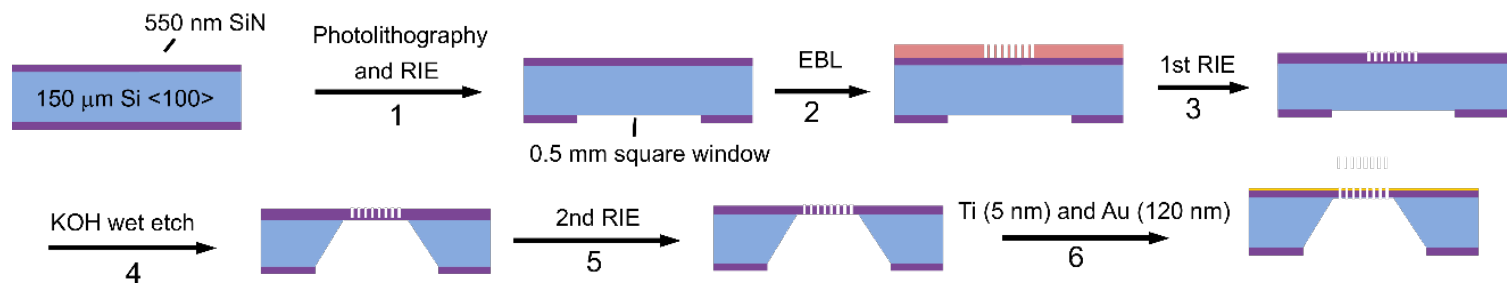

Scheme 1. Workflow of the fabrication process. 1) A Si window was opened at the back of the wafer by a standard photolithography and RIE; 2-3) The NHA structures were produced at the front of the wafer using EBL and transferred to the SiN surface by another RIE; 4) The exposed Si window at the back was wet etched with $\mathrm{KOH}$; 5) The remaining SiN layer was etched by another RIE from the back; 6) The NHA structures were finally deposited with $5 \mathrm{~nm}$-Ti and $120 \mathrm{~nm}$-Au. (RIE: reaction ion etching; EBL: E-beam lithography; KOH: Potassium hydroxide) 

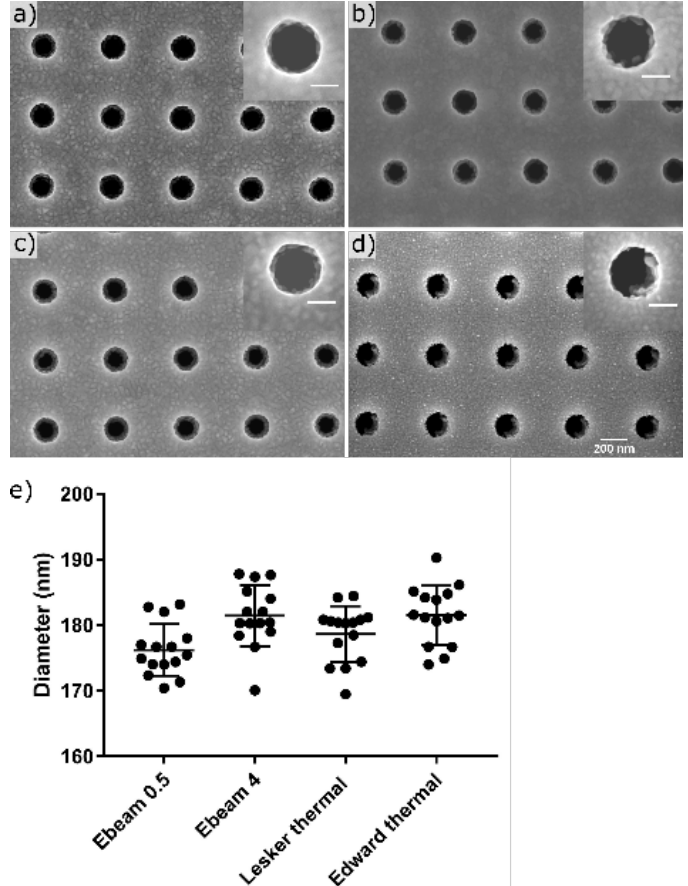

Fig. 1 SEM images and analysis of NHA via different metal deposition

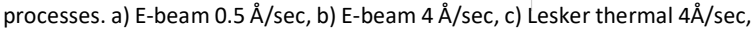
d) Edward thermal $4 \AA$ $/ \mathrm{sec}$. Scale bar for all figures is shown in d): $200 \mathrm{~nm}$. Inset scale bar: $100 \mathrm{~nm}$. e) Hole diameter comparison from four processes.

sample liquids can flow through the surface and lead to an improved mass transport as well as analyte concentrating on the surface. ${ }^{32,33}$ Second, the through-hole structure allows the detection in both reflection and transmission modes, which has the potential to be developed into a portable and image-based readout system. ${ }^{17}, 34$ Third, though most nanoplasmonic structures are implemented on transparent materials such as glass, the high refractive index of SiN provides a well-defined sharp resonance peak in the transmission/reflectance spectrum. ${ }^{23}$ To achieve this through-hole structure on suspended SiN film, a Si window was first opened to define the sensing site at the back of the wafer by a standard photolithography and reactive ion etching (RIE) (Step 1). The NHA structures were produced at the front of the wafer using E-beam lithography (EBL, Step 2) and transferred to the SiN surface by another RIE (Step 3). In this step, only a partial SiN layer was etched..$^{35}$ The purpose of this partial etch is that a complete SiN etched at the front has been observed to lead to cracking issues in the subsequent $\mathrm{KOH}$ wet etch process step. Thus, the remaining SiN can protect the front Si surface from the subsequent $\mathrm{Si}$ etching with $\mathrm{KOH}$. The exposed Si window at the back was wet etched with $\mathrm{KOH}$ subsequently, so that the $\mathrm{Si}$ region was removed, and suspended SiN was formed (Step 4). The remaining SiN layer was etched by another RIE from the back to create the through-hole NHA structures (Step 5). The NHA structures were finally deposited with $5 \mathrm{~nm}-\mathrm{Ti}$ and $120 \mathrm{~nm}$ Au to render the surface with plasmonic properties.

Further, we utilised four different evaporation processes to evaluate the influence of the metal film qualities to the NHA sensing performance. The four deposition processes are detailed in the Method section, namely Ebeam 0.5 and Ebeam 4 using two different deposition rates $0.5 \AA / \mathrm{sec}$ and $4 \AA / \mathrm{sec}$ in E-beam evaporation and Lesker thermal and Edward thermal using two different thermal evaporation equipment at the same rate of $4 \AA$ $/ \mathrm{sec}$.

In this work, we used a defined hole size (diameter $100 \mathrm{~nm}$ during $\mathrm{EBL}$ design) and periodicity (hole centre-to-centre distance $530 \mathrm{~nm}$ ) as the model to evaluate the influence of metal quality to the sensing performance. The determination of this optimised hole diameter and periodicity is based on our investigation in the influence of hole size and periodicity to the optical reflectance spectrum and sensitivity (Fig. S1, ESI+). As the hole morphology was kept the same in the same fabrication process, the influence was only caused by the metal -related morphologies from different metal deposition processes.

Subsequently, the surface topography (i.e. surface roughness) information was obtained by AFM, then surface morphology and grain morphology were analysed by SEM and SEM-based EBSD, respectively. Using this approach, i.e., measuring roughness prior to grain morphology, we can minimize carbon contamination and sample damage caused by SEM.

To address imaging surface structures, SEM and AFM have complementary capabilities. SEM have fantastic depth of field and is capable of imaging structures that have a strong vertical relief. AFM's have poor depth of field, but provide better contrast on flat samples or surface features compared to SEM.

\section{Surface Morphology by SEM}

Fig. 1 presents the SEM images of the NHA structures after metal deposition, which shows well-defined hole structures. Although the designed hole diameter was $100 \mathrm{~nm}$ during EBL, the actual holes were larger considering the RIE process is isotropic with both lateral and vertical etching. The hole sizes after four different metal deposition processes have no significant differences (Fig. 1e). Therefore, the differences in sensing performance (which will be discussed in later sections) are not due to the influence of the hole size or periodicity. From zoom-in images of each individual holes, it can be seen that there were some side depositions on the inside walls of the nanoholes from all deposition methods which is inevitable. However, the side depositions from different methods are slightly different (more SEM images are provided in Fig. S2, $\mathrm{ESI}^{+}$). During these methods, Ebeam 0.5 provided a slightly smoother inner wall surface compared to Ebeam 4 and Lesker thermal which have rougher inner wall surface with larger metal clusters. Ebeam 4 and Lesker thermal have similar morphology in the inner wall surface considering they are using the same deposition rate ( $4 \AA / \mathrm{sec})$. Interesting, Edward thermal provided an asymmetric inner surface deposition that partial inner surface was covered with Au (Fig. 1d). There are some fluctuations encountered when we used the Edward evaporator 


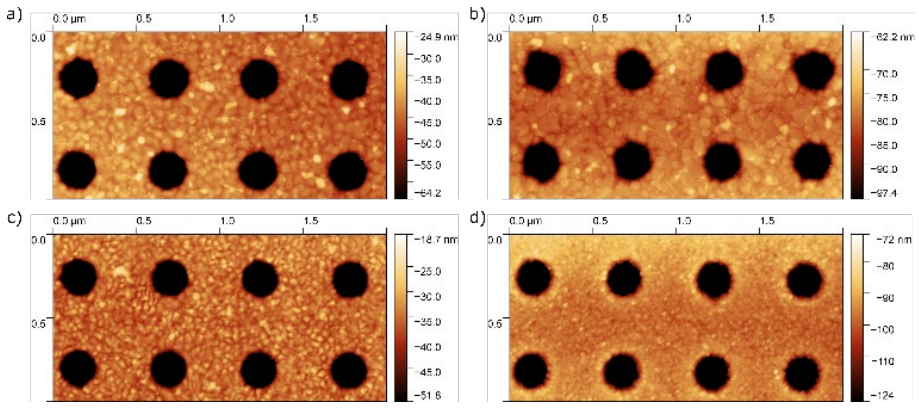

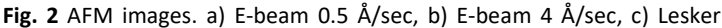
thermal $4 \AA \AA$ sec, d) Edward thermal $4 \AA$ $/$ sec.

(Table S1 ESI†, from $6.5 \times 10^{-6}$ to $1.5 \times 10^{-5}$ Torr) and it could be correlated. There may be some shadowing effect from the relative position of the target to the sample to make the deposition appear asymmetric.

Although SEM provides surface morphology, the surface topography (i.e. surface roughness) and grain morphology can be precisely measured by AFM and SEM-based EBSD. The results are shown in Fig. 2 and 3 with quantitative summary in Table 1.

\section{Surface Topography by AFM}

AFM provides more fine details about the surface roughness compared to SEM (Fig 2.). Within E-beam depositions methods, while the slower gold deposition rate (Ebeam 0.5 ) provided a smoother inner wall as seen from SEM images, it produced marginal higher roughness compared to faster deposition rate $4 \AA / \mathrm{sec}$ (Ebeam 4). This is correlated with the larger grain size from Ebeam 0.5 (Table 1), as larger grain size provides higher peaks in topography that leads to higher surface roughness. On the other hand, within thermal depositions methods, Lesker produced smoother surface compared to Edward while keeping the same deposition rate of $4 \AA / \mathrm{sec}$. This could again be attribute to the sudden fluctuations in the base pressure with Edward instrument. Interestingly, when comparing Lesker thermal with E-beam 4 (both at same $4 \AA$ A $/ \mathrm{sec}$ ), it can be noticed that former forms smoother surface that can be read from the RMS roughness in Table 1. This result indicates that even the deposition flow-rate and base pressure are kept same, other key factors such as the methodology the source material is evaporated (via E-beam bombardment or thermal heating) can influence the surface morphology. Unfortunately, inner wall morphology could not be obtained by AFM because of the limitation in the geometry and aspect ratio of AFM tip we used, which created artificial images when measuring the vertical side wall. Overall, comparison of the AFM results Table 1 demonstrates that that the Lesker thermal $4 \AA$ A $/$ sec produces the smoothest surface compared to other deposition methods, which is necessary criteria for better sensing performance and this will be discussed later.

\section{Grain Morphology by SEM-EBSD}

Next, the grain morphology was obtained via SEM-based EBSD. SEM-based EBSD techniques have a significant advantage over a)
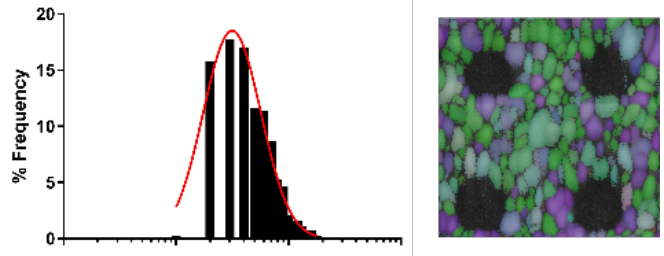

b)
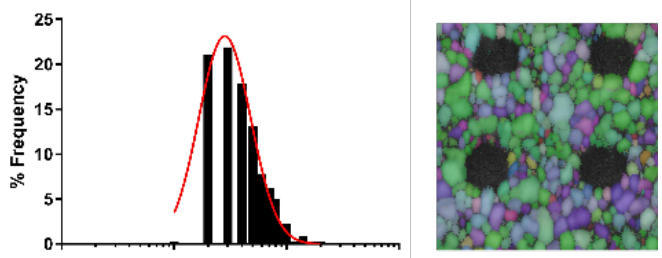

c)
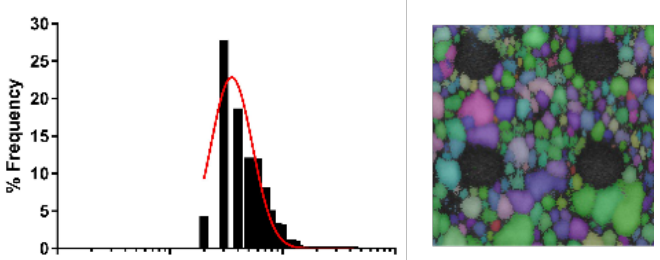

d)
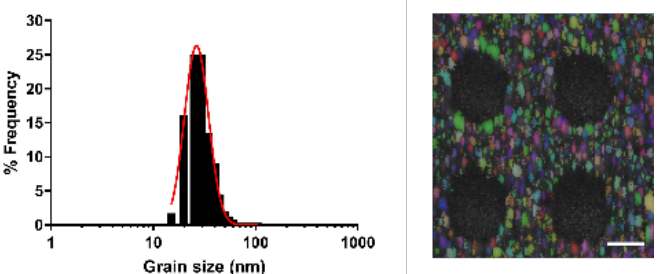

Fig. 3 Grain size distribution and EBSD images. a) E-beam $0.5 \AA / \mathrm{sec}, \mathrm{b}$ ) $\mathrm{E}$ -

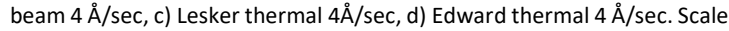
bar: $200 \mathrm{~nm}$.

other counterparts in automated analysis of grain morphology with diffraction systems. ${ }^{36,} 37$ Automated analysis of grain morphology is inevitable because grains and their grain boundaries occupy a considerable volume in the NTA structures. The technique can provide a spatial resolution of 30$100 \mathrm{~nm}$ with key nanostructural information, such as orientation mapping, grain size analysis and pole figures.

The grain size distributions are shown in Fig 3. All the grain size distributions from four samples followed a good lognormal distribution. The geometric mean after lognormal fitting was used to compare the grain sizes of four samples. Edward thermal provided the smallest grain size with a narrowest size distribution, while Ebeam 0.5 provided the largest grain size with the broadest size distribution. The large grain size provided by the lower evaporation rate $0.5 \AA / \mathrm{sec}$ is because there is more time for freshly added metal atoms to diffuse on the substrate

Table 1. Quantitative surface roughness and grain size from AFM and EBSD.

\begin{tabular}{|c|c|c|}
\hline Sample & $\begin{array}{c}\text { RMS roughness } \mathbf{S}_{\mathbf{q}} \\
(\mathbf{n m})\end{array}$ & $\begin{array}{c}\text { Grain size } \mathbf{d}_{\text {mean }} \text { (Geometric } \\
\text { mean } \pm \text { standard deviation, } \mathbf{n m} \text { ) }\end{array}$ \\
\hline Ebeam 0.5 & 2.93 & $44.5 \pm 1.8$ \\
\hline Ebeam 4 & 2.58 & $37.4 \pm 1.7$ \\
\hline Lesker & 2.30 & $42.2 \pm 1.5$ \\
\hline Edward & 4.06 & $28.5 \pm 1.3$ \\
\hline
\end{tabular}

before being bombarded by additional atoms, so that grains 

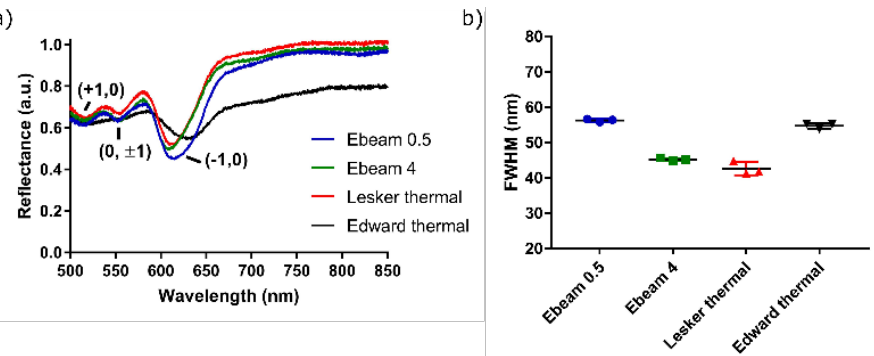

Fig. 4 Reflectance spectra results. a) Reflectance spectra from four deposition processes. As three measurements from one deposition process are very similar, only one spectrum was shown for each method. b) The calculated FWHM from each sample showing Ebeam 4 and Lesker thermal provide smaller FWHM, while Ebeam 0.5 and Edward thermal provide larger FWHM.

have more time to grow. ${ }^{30}$ Interestingly, from Table 1 it can be seen that the surface roughness and grain size does not have a certain pattern of relationship. This is because the roughness depends not only on the grain size but also the shape and orientation of the grains.

\section{Sensing performance of the NHA senso}

As the NHA presents EOT, more light will be transmitted through the holes near the EOT resonant wavelength, which indicates less light is reflected near the resonant wavelength. In our study, we measured reflectance spectrum for the readout. We used key parameters in biosensing including the line withFull Width at Half Maximum (FWHM) in resonance peak and bulk sensitivity to evaluate the sensing performance.

Fig. 4a shows the reflectance spectra of the four samples measured in air, all of which show a prominent dip around 610 $\mathrm{nm}$, which is corresponding to the $(-1,0)$ grating order (hereinafter referred to as the peak position). There are also two other modes at around $550 \mathrm{~nm}$ and $510 \mathrm{~nm}$, corresponding to the $(0, \pm 1)$ and $(+1,0)$ grating orders. The appearance of these modes are consistent with similar NHA structures that have been reported before. ${ }^{38}$ Each of the four samples was measured over three replicate sensing sites, and the spectra of the three replicates are very similar, providing very close peak positions (Fig. S3, ESI+) and FWHM (Fig. 4b). This indicates that our fabrication process is very reproducible. It can be seen from Fig. S3 that Edward thermal provided a larger peak position around $630 \mathrm{~nm}$, while the other three methods provided similar peak position around $610 \mathrm{~nm}$. Considering that the four methods provide NHA with similar hole sizes (shown in Fig. 1e) and same periodicity, we attribute the large deviation in peak position with Edward Thermal to the asymmetric inner surface deposition

During spectrum-based sensing, an important parameter to evaluate the sensing is the line width FWHM. The narrow the peak the better signal to noise ratio that can be achieved, allowing smaller changes to be detected assuming that the detection accuracy is inversely proportional to FWHM. ${ }^{39}$ Among the four samples, Ebeam 4 and Lesker thermal provided very similar and the smaller FWHM: $45.2 \mathrm{~nm}$ and $42.6 \mathrm{~nm}$ in average while Ebeam 0.5 and Edward thermal provided larger FWHM: $56.2 \mathrm{~nm}$ and $54.7 \mathrm{~nm}$ in average (Fig. 4b). This difference indicates that the line width is influenced by the metal surface morphology.
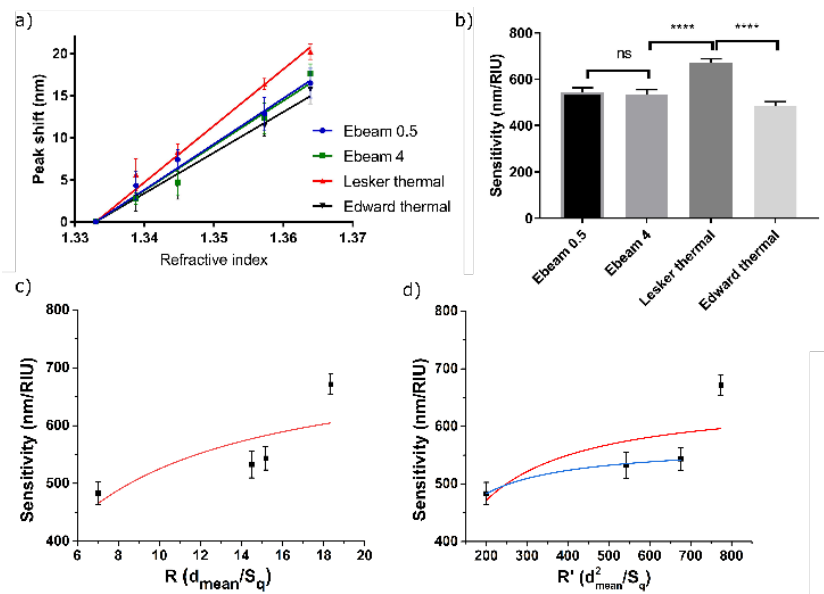

Fig. 5 Sensitivity test $(n=3)$. a) The relationships between resonance peak shifts and refractive index from four samples. b) Comparison of the sensitivity from the four samples. Comparison was made by One-way ANOVA with Tukey's test. Detailed comparison is as follows. Ebeam 0.5 vs Ebeam 4: No Significant (ns), $\mathrm{p}=0.8299$; Ebeam 0.5 vs Lesker thermal: Significant $\left({ }^{* * * *}\right), \mathrm{p}<0.0001$; Ebeam 0.5 vs Edward thermal: Significant $\left({ }^{* *}\right), p=00012$; Ebeam 4 vs Lesker thermal: Significant $\left({ }^{* * *}\right), \mathrm{p}<0.0001$; Ebeam 4 vs Edward thermal: Significant $\left({ }^{* *}\right)$, $\mathrm{p}=0.0065$; Lesker thermal vs Edward thermal: Significant $\left({ }^{* * * *}\right), p<0.0001$. c-d) empirical relationship between the metal film structural morphology (using $R$ and $R^{\prime}$ respectively for $c$ and $d$ ) and bulk sensitivity.

Subsequently, the bulk sensitivities of the NHA samples were evaluated by immersing them in a series of solutions with different refractive index. As a refractive index-based sensor, an increase of refractive index surrounding the NHA film leads to a red shift in the reflectance spectrum. The relationships between resonance peak shifts and refractive index from four samples were plotted in Fig. 5a, and the comparison of the sensitivity was shown in Fig. $5 b$.

To correlate the differences in sensitivity with film quality, an empirical equation was deduced using grain size and surface roughness to express the sensor sensitivity. In a periodic nanohole arrays, the phase-matching condition for arising of SPR excitation peaks coincides with the Bragg resonances of the ordered structures ${ }^{40}$. Thus, for a square array of nanoholes, the EOT resonant wavelength, $\lambda_{\text {(SP) }}$ can be estimated as,

$$
\lambda_{(S P)}=\frac{a_{0}}{\sqrt{i^{2}+j^{2}}} \sqrt{\frac{\varepsilon_{d} \varepsilon_{m}}{\varepsilon_{d}+\varepsilon_{m}}}
$$

where $a_{0}$ is the lattice constant of the array, $i$ and $j$ are integers defining the scattering orders of the square array, and $\varepsilon_{\mathrm{d}}$ and $\varepsilon_{\mathrm{m}}$ is the real part of the relative permittivity of the Au metal and the surrounding solution medium ${ }^{40}$. As shown in Fig. $5 \mathrm{a}$, the refractive index sensitivity (RIS) is defined as resonance peak shifts $(D \lambda)$ divided by change of refractive index $(D n$, or $D \varepsilon)$. Namely, the RIS can be expressed as, 


$$
\begin{gathered}
R I S=\frac{\Delta \lambda}{\Delta \varepsilon_{d}}=\frac{d \lambda_{S P}}{d \varepsilon_{d}}=\frac{a_{0}}{2 \sqrt{i^{2}+j^{2}}} \sqrt{\frac{\varepsilon_{m}^{3}}{\varepsilon_{d}\left(\varepsilon_{m}+\varepsilon_{d}\right)^{3}}}= \\
\frac{a_{0}}{2 \sqrt{i^{2}+j^{2}}} \frac{1}{\sqrt{\varepsilon_{d}}}\left(\frac{1}{1+\frac{\varepsilon_{d}}{\varepsilon_{m}}}\right)^{\frac{3}{2}}
\end{gathered}
$$

Considering the same set of solutions were used as the surrounding medium to test the sensor sensitivity, $\varepsilon_{\mathrm{d}}$ can also be seen as a constant in our system. In this case, RIS only depends on $\varepsilon_{\mathrm{m}}$, which is further determined by the Au metal film quality. Ideally, $\varepsilon_{m}$ reaches the maximum value when an ultra-smooth metal film (infinite grain size and nearly " 0 " roughness) is present, and result is the optimum RIS based on Eqn (2). However, the practical value of $\varepsilon_{m}$ is usually smaller due to extra scattering or diffusion paths for incident photons which are caused by the surface defects in the metal film. Given smaller grain sizes and larger surface roughness will introduce more surface defects and thus reduce the permittivity of metal films, $\varepsilon_{m}$ is in positive correlation with grain size $\left(d_{\text {mean }}\right)$ while in negative correlation with surface roughness $\left(\mathrm{S}_{\mathrm{q}}\right)$.

To investigate how the intrinsic properties of the metal film influence the sensor RIS, a parameter "R" was introduced to define the smoothness of the metal film, and $\varepsilon_{m}$ can be semiempirically expressed as,

$$
\varepsilon_{m}=k R \quad \text { (3) }
$$

where $\mathrm{k}$ is introduced as a constant to assume that $\varepsilon_{\mathrm{m}}$ is in simplest linear relationship with the defined parameter $\mathrm{R}$. In the most simplified case, $\mathrm{R}=\mathrm{d}_{\text {mean }} / \mathrm{S}_{\mathrm{q}}$, and

$$
\varepsilon_{m}=k d_{\text {mean }} / S_{q}(4)
$$

Therefore, RIS is semi-empirically expressed by $d_{\text {mean }}$ and $S_{q}$ by taking Eqn (4) to Eqn (2), which is further employed to fit the experimental "RIS VS $\mathrm{d}_{\text {mean }} / \mathrm{S}_{\mathrm{q}}$ " curve as shown in Fig. 5c. Despite some discrepancy to individual experimental data point, the proposed model can correctly estimate the overall trend of RIS changing with the film quality defined by $d_{\text {mean }} / S_{q}$ Considering the grain size (area) is a 2-Dimention parameter, it is reasonably using an optimized $\mathrm{R}^{\prime}=\mathrm{d}^{2}$ mean $/ \mathrm{S}_{\mathrm{q}}$ in the proposed model, which gives,

$$
\varepsilon_{m}=k^{\prime} d_{\text {mean }}^{2} / S_{q} \quad \text { (5) }
$$

Eqn (2\&5) were applied to the experimental "RIS VS d ${ }^{2}$ mean $/ \mathrm{S}_{\mathrm{q}}$ " curve again to examine the validity of the proposed model, as shown in Fig. $5 \mathrm{~d}$. The overall fitting didn't show considerable improvement comparing with the one using $\mathrm{R}$ (red trending lines). However, the modified model accurately matches the experimental in the range where $\mathrm{R}^{\prime}<700$. It means our modified model can make an almost accurate estimation for the RIS for the rougher film surfaces (blue line).

Among the four deposition methods, Lesker thermal provided the highest sensitivity $671.3 \pm 17.4 \mathrm{~nm} / \mathrm{RIU}$, while Edward thermal provided the lowest sensitivity $483.3 \pm 19.4 \mathrm{~nm} / \mathrm{RIU}$. Ebeam 0.5 and Ebeam 4 provided similar sensitivities $543.4 \pm$ $20.15 \mathrm{~nm} /$ RIU and $532.6 \pm 23.1 \mathrm{~nm} / \mathrm{RIU}$ which were lower than Lesker thermal and higher than Edward thermal. Here, we provided an empirical relationship between the sensitivity and the grain sizes (average grain diameter, $d_{\text {mean }}$ ) and surface roughness (RMS roughness, $S_{q}$ ) to evaluate their influence on the sensitivity based on their ratios $R=D_{\text {mean }} / S_{q}$ as both the two parameters contribute. It can be seen from Fig. $5 c$ that a larger $\mathrm{R}$ (indicating a larger grain size and smoother surface) led to a higher sensitivity. In our work, Lesker thermal had the largest ratio $\mathrm{R}$ and correspondingly the best sensitivity, while Edward thermal had the worst sensitivity as it has the smallest $R$. Ebeam 0.5 and Ebeam 4 had very similar $R$ and their sensitivities were very similar (Fig. $5 \mathrm{c}$ ). As both surface roughness and grain boundaries limit surface-plasmon propagation, smooth surface with few grain boundaries (i.e. larger grains) are preferred for plasmonic sensor, thus larger grains and smoother surface can lead to better sensing performance. This is consistent with the work by Nagpal et al. that single crystalline with ultrasmooth surface can provide high-quality plasmonic materials and that by Zhang et al. demonstrating a thermal annealing can lead to a smoother surface and thus improve the sensitivity of NHA sensors. ${ }^{26,} 27$ Interestingly, the sensitivity and line width in Fig. $4 \mathrm{~b}$ does not have a clear relationship. For instance, Ebeam 0.5 had the largest FWHM but it does not necessarily have the worst sensitivity. Thus, the line width seems not have a direct indication for the actual sensitivity as it is correlated to the detection accuracy. In addition, the asymmetric inner wall deposition from Edward thermal may also contribute to its low sensitivity.

\section{Conclusions}

In this work, we fabricated NHA structures along with several different metal deposition processes. We characterised the metal film quality around the holes using several nondestructive nanoscale surface characterisations including SEM, $A F M$ and EBSD and obtained quantitative and comparative analysis of Au quality of our fabricated NHA sensor. Among these deposition methods, Edwards Auto500 thermal evaporator cannot provide a satisfactory film quality because it provided an asymmetric inner wall deposition. Quantitative results about surface roughness and grain size were obtained and we demonstrated an empirical relationship regarding their influence on the sensing performance. Specifically, the ratio of grain size and roughness was used to evaluate the bulk sensitivity - the sensitivity was higher when the ratio was larger. Judging from these criteria, Lesker Thermal $4 \AA$ A $/ \mathrm{sec}$ provided the best optical performance in term of narrower spectrum line width and higher sensitivity because of a good combination of grain size and surface roughness. We successfully correlated the sensor sensitivity with the film quality with a semi-empirical model by introducing a parameter R. In this specific way, we obtained a unique insight into the quality of the deposited $\mathrm{Au}$ film and established an empirical criterion for evaluating and optimising our fabricated NHA plasmonic sensor for better sensing performance in the future. However, due to the complicated scattering or diffusion paths for incident photons caused by poor film quality, it is very challenging to develop an accurate model to estimate the RIS of fabricated nano-hole arrays. The proposed model in this report is just a first step towards that meaningful milestone. 


\section{Method}

Fabrication of plasmonic NHA. Scheme 1 illustrates the procedure for fabricating NHA structure. A 4-inch silicon wafer (150 $\mu \mathrm{m}$ thick) with a 550nm silicon nitride (SiN) deposited by low pressure chemical vapor deposition was purchased from Virginia Semiconductor, USA. To fabricate NHA structures on a suspended SiN film, a Si window was first opened to define the sensing site at the back of the wafer by a standard photolithography with AZ6632 positive resist, and transferred to $\mathrm{SiN}$ by reactive ion etching (RIE) with $\mathrm{CF}_{4} 20 \mathrm{sccm}, \operatorname{Ar} 65 \mathrm{sccm}$, chamber pressure $75 \mathrm{mTorr}$ and radio frequency power $100 \mathrm{~W}$. The NHA structures were produced at the front of the wafer using E-beam lithography (EBL) with AR-P 6200.13 E-beam resist (Allresist). The designed structure in EBL was hole diameter 100 $\mathrm{nm}$ with centre-to-centre distance $530 \mathrm{~nm}$. The structure was transferred to the SiN layer by another RIE with $\mathrm{CF}_{4} 30 \mathrm{sccm}$, $\mathrm{CHF}_{3} 20 \mathrm{sccm}$, chamber pressure $25 \mathrm{mTorr}$ and radio frequency power $100 \mathrm{~W}$. In this step, only a partial SiN layer was etched to protect the front Si surface from the subsequent Si wet etching. The exposed Si window at the back was wet etched with $45 \%$ $w / v \mathrm{KOH}$ aqueous solution $\mathrm{KOH}$ at $85^{\circ} \mathrm{C}$, so that Si region was removed, and suspended SiN was formed. The remaining SiN layer was etched by another RIE from the back to create the through-hole NHA structures.

Metal deposition. After the NHA structures was created in a single process, the wafer was cleaved into four identical pieces and deposited with $5 \mathrm{~nm} \mathrm{Ti}$ (to improve the adhesion) and 120 $\mathrm{nm}$ gold by four evaporation processes respectively. Two samples were deposited with e-beam evaporation using one PVD75 e-beam evaporator (Kurt J. Lesker) at two different deposition rates: $0.5 \AA / \mathrm{sec}$ and $4 \AA / \mathrm{sec}$. The samples were hereinafter referred to as Ebeam $\mathbf{0 . 5}$ and Ebeam 4. Another two samples were deposited with two different thermal evaporation equipment: Lesker thermal evaporator and Edwards Auto500 thermal evaporator at the same rate of 4 $\AA / \mathrm{sec}$. The samples were hereinafter referred to as Lesker thermal and Edward thermal. The detailed instruments conditions are summarized in Table. S1.

Nanoscale surface characterisations. The fabricated NHA was imaged directly using FEI Nova NanoSEM 450. The hole diameter was measured by ImageJ software using the outer edge of the nanohole encountering the planar gold surface. The surface topography of the Au layer was examined over $2 \times 1 \mu \mathrm{m}^{2}$ hole regions using AFM (Bruker Dimension Icon). Peak force tapping mode with the SCANASYST probe (from Bruker AFM probes) was used to perform the measurements. AFM images were analysed using Gwyddion 2.53 software. The root-meansquared (RMS) surface roughness was measured over the entire image exclusive of the hole regions. Surface morphology (including grain size, grain boundaries and crystallographic orientation) was investigated by a Field Emission Gun-SEM (Carl Zeiss ULTRA Plus) equipped with a newly developed revolutionary CMOS (EBSD) detector. The data analysis was performed using Oxford Aztec processing software. The grain sizes were presented using the Maximum Feret Diameter. Grain size distribution was plotted and analysed using Prism 7.04, and the geometric mean after the lognormal fitting was using to compare the grain sizes of four samples.

Optical reflectance spectrometry. Optical reflectance spectra were measured as described in our previous work. ${ }^{41,} 42$ The spectra were measured in the visible and near infrared at normal incidence using a custom-built optical arrangement. The setup incorporated a USB2000+ miniature fibre-optic spectrometer (Ocean Optics Inc.) and a fibre-coupled light source (Mikropack GmbH, Germany). The optical arrangement was incorporated with a 2-axis $(X-Y)$ automated stage (MS2000, Applied Scientific Instrumentation). A custom software platform, driven by LabVIEW (National Instruments, TA), was used to process the spectra and provide a program for automatically moving of the stage. The peak position of each reflectance spectrum was gathered by recording the wavelength corresponding to the maximum intensity after a polynomial fitting of the peak. The full width at half maximum (FWHM) of the peak was calculated using OriginPro 9 after a Gaussian fitting of the $(-1,0)$ mode (Fig. S4, ESI ${ }^{+}$). The sensitivity test was performed by immersing the samples into different concentrations of sucrose/water solutions (\% w/w), which provides solutions with defined refractive index as below: $0 \%$ 1.3330, 4\%-1.3388, 8\%-1.3448, 16\%-1.3573, 20\%-1.3639. The sensitivity (peak shift per refractive index unit) was obtained using the slope after a linear fitting of the plots of refractive index against peak shift. The comparison of the sensitivity from four methods was performed by one-way ANOVA with Tukey's test in Prism.

\section{Conflicts of interest}

There are no conflicts to declare.

\section{Acknowledgements}

This research was funded by Cancer Institute NSW Early Career Fellowship (2017/EFC012, YZ), Avner Pancreatic Cancer Foundation Grant (www.avnersfoundation.org.au) and Ovarian Cancer Research Foundation (GA-2019-13). We thank Professor Adam Micolich and his group from School of Physics, UNSW Sydney for providing support for Edward Edwards Auto500 thermal evaporator. We thank Australian National Fabrication Facility-NSW Node for the training and support for the fabrication processes. We thank Electron Microscope Unit from UNSW Mark Wainwright Analytical Centre and the Australian Centre for Microscopy and Microanalysis from The University of Sydney for all the training and support for the characterisations. We thank Dr Hyungsoon Im from Center for Systems Biology, Massachusetts General Hospital for helpful discussions about the fabrication of NHA sensor.

\section{References}


1. S. M. Borisov and O. S. Wolfbeis, Chemical Reviews, 2008, 108, 423-461.

2. Nanotechnology in Australia: trends, applications and collaborative opportunities, Australian Academy of Science, 2009.

3. M. A. Cooper, Nature Reviews Drug Discovery, 2002, 1, 515-528.

4. A. G. Brolo, Nat Photon, 2012, 6, 709-713.

5. M. C. Estevez, M. A. Otte, B. Sepulveda and L. M. Lechuga, Analytica Chimica Acta, 2014, 806, 55-73.

6. A. Abbas, M. J. Linman and Q. Cheng, Biosensors and Bioelectronics, 2011, 26, 1815-1824.

7. S. S. Hinman, K. S. McKeating and Q. Cheng, Analytical Chemistry, 2018, 90, 19-39.

8. H. Nguyen, J. Park, S. Kang and M. Kim, Sensors-Basel, 2015, 15, 10481.

9. K. A. Willets and R. P. Van Duyne, Annual Review of Physical Chemistry, 2007, 58, 267-297.

10. K. M. Mayer and J. H. Hafner, Chemical Reviews, 2011, 111 3828-3857.

11. M. E. Stewart, C. R. Anderton, L. B. Thompson, J. Maria, S. K. Gray, J. A. Rogers and R. G. Nuzzo, Chemical Reviews, 2008, 108, 494-521.

12. N. C. Lindquist, P. Nagpal, K. M. McPeak, D. J. Norris and S.H. Oh, Reports on Progress in Physics, 2012, 75, 036501.

13. A. Prasad, J. Choi, Z. Jia, S. Park and M. R. Gartia, Biosensors and Bioelectronics, 2019, 130, 185-203.

14. O. Tokel, F. Inci and U. Demirci, Chemical Reviews, 2014, 114, 5728-5752.

15. T. W. Ebbesen, H. J. Lezec, H. F. Ghaemi, T. Thio and P. A. Wolff, Nature, 1998, 391, 667-669.

16. A. Belushkin, F. Yesilkoy and H. Altug, ACS Nano, 2018, 12, 4453-4461.

17. A. E. Cetin, A. F. Coskun, B. C. Galarreta, M. Huang, D. Herman, A. Ozcan and H. Altug, Light: Science \& Applications, 2014, 3.

18. F. Yesilkoy, R. A. Terborg, J. Pello, A. A. Belushkin, Y. Jahani, V. Pruneri and H. Altug, Light: Science \& Applications, 2018, 7, 17152.

19. J. Kim, Lab on a Chip, 2012, 12, 3611-3623.

20. F. Eftekhari, C. Escobedo, J. Ferreira, X. Duan, E. M. Girotto, A. G. Brolo, R. Gordon and D. Sinton, Analytical Chemistry, 2009, 81, 4308-4311.

21.

K. Xiong, G. Emilsson and A. B. Dahlin, Analyst, 2016, 141, 3803-3810.

22. T. Ohno, C. Wadell, S. Inagaki, J. Shi, Y. Nakamura, S. Matsushita and T. Sannomiya, Opt. Mater. Express, 2016, 6, 1594-1603.

23. A. E. Cetin, D. Etezadi, B. C. Galarreta, M. P. Busson, Y. Eksioglu and H. Altug, ACS Photonics, 2015, 2, 1167-1174.

24. E. S. P. Leong, Y. J. Liu, B. Wang and J. Teng, ACS Applied Materials \& Interfaces, 2011, 3, 1148-1153.

25. J.-C. Tinguely, I. Sow, C. Leiner, J. Grand, A. Hohenau, N. Felidj, J. Aubard and J. R. Krenn, BioNanoScience, 2011, 1, 128-135.

26.

P. Nagpal, N. C. Lindquist, S.-H. Oh and D. J. Norris, Science, 2009, 325, 594-597.

27. J. Zhang, M. Irannejad, M. Yavuz and B. Cui, Nanoscale Research Letters, 2015, 10, 238.

28. R. D. Averitt, S. L. Westcott and N. J. Halas, J. Opt. Soc. Am. $B, 1999,16,1824-1832$.
29.

30.

31.

32.

33.

34.

35.

K. S. Yang, H. Im, S. Hong, I. Pergolini, A. F. del Castillo, R. Wang, S. Clardy, C.-H. Huang, C. Pille, S. Ferrone, R. Yang, C. M. Castro, H. Lee, C. F. del Castillo and R. Weissleder, Science Translational Medicine, 2017, 9.

36. M. A. Khan, H. Chen, J. Qu, P. W. Trimby, S. Moody, Y. Yao, S. P. Ringer and R. Zheng, ACS Applied Materials \& Interfaces, 2017, 9, 24259-24272.

37. M. A. Khan, P. Bian, J. Qu, H. Chen, H. Liu, M. Foley, Y. Yao, S. P. Ringer and R. Zheng, Ultramicroscopy, 2019, 196, 1-9.

38. A. A. Yanik, A. E. Cetin, M. Huang, A. Artar, S. H. Mousavi, A. Khanikaev, J. H. Connor, G. Shvets and H. Altug, Proceedings of the National Academy of Sciences, 2011, 108, 11784-11789.

39. A. K. Sharma, R. Jha and H. S. Pattanaik, Journal of Applied Physics, 2010, 107, 034701.

40. R. Gordon, D. Sinton, K. L. Kavanagh and A. G. Brolo, Accounts of Chemical Research, 2008, 41, 1049-1057.

41. Y. Zhu, A. H. Soeriyadi, S. G. Parker, P. J. Reece and J. J. Gooding, Journal of Materials Chemistry B, 2014, 2, 35823588.

42. Y. Zhu, B. Gupta, B. Guan, S. Ciampi, P. J. Reece and J. J. Gooding, ACS Applied Materials \& Interfaces, 2013, 5, 6514-6521. 